\title{
Standardization of surface potential measurements of graphene domains
}

SUBJECT AREAS:

ELECTRONIC PROPERTIES AND DEVICES

ELECTRONIC DEVICES

IMAGING TECHNIQUES

MICROSCOPY

Received

15 May 2013

Accepted

12 August 2013

Published

6 September 2013

Correspondence and requests for materials should be addressed to O.K. (olga.kazakova@ npl.co.uk)

\author{
Vishal Panchal ${ }^{1,2}$, Ruth Pearce', Rositza Yakimova ${ }^{3}$, Alexander Tzalenchuk ${ }^{1,2}$ \& Olga Kazakova'
}

\author{
${ }^{1}$ National Physical Laboratory, Teddington, TW 11 OLW, UK, ${ }^{2}$ Royal Holloway, University of London, Egham, TW20 OEX, UK, \\ ${ }^{3}$ Linköping University, Linköping, S-581 83, Sweden.
}

We compare the three most commonly used scanning probe techniques to obtain a reliable value of the work function in graphene domains of different thickness. The surface potential (SP) of graphene is directly measured in Hall bar geometry via a combination of electrical functional microscopy and spectroscopy techniques, which enables calibrated work function measurements of graphene domains in ambient conditions with values $\Phi_{1 L G} \sim 4.55 \pm 0.02 \mathrm{eV}$ and $\Phi_{2 L G} \sim 4.44 \pm 0.02 \mathrm{eV}$ for single- and bi-layer, respectively. We demonstrate that frequency-modulated Kelvin probe force microscopy (FM-KPFM) provides more accurate measurement of the SP than amplitude-modulated (AM)-KPFM. The discrepancy between experimental results obtained by different techniques is discussed. In addition, we use FM-KPFM for contactless measurements of the specific components of the device resistance. We show a strong non-Ohmic behavior of the electrode-graphene contact resistance and extract the graphene channel resistivity.

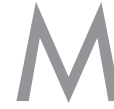
apping of the local electronic properties of graphene is necessary for control of growth parameters and for understanding device functionality. The accurate quantification of the measured values is essential in order for the properties of graphene to be reliably understood and compared.

The growth of graphene by the sublimation of $\mathrm{Si}$ from $\mathrm{SiC}$ is arguably the most advanced method for producing continuous, homogeneous large area graphene ${ }^{1}$. Control over the layer thickness has been demonstrated ${ }^{2}$, with sublimation being the method of choice for device manufacture, where a continuous, large area of single-layer (1LG) of graphene is required, i.e. nanoelectronics, sensing, $\mathrm{THz}$ applications, etc. Due to advances in the sample growth, it is generally possible to achieve homogenous single layer coverage over large areas (i.e. 95\% 1LG coverage for the sample presented in this work). However, even small inclusions of bi-layer graphene (2LG) leads to redistribution of carriers, inhomogeneous screening effects, and the corresponding nanoscale changes in the surface potential (SP) and work function $(\Phi)$. Unambiguous determination of the layer thickness of epitaxially grown graphene using atomic force microscopy (AFM) is particularly challenging due to the stepped nature of the $\mathrm{SiC}$ substrate coupled with growth of graphene, which often nucleates at step edges ${ }^{1}$.

Scanning measurement techniques, such as Kelvin probe force microscopy (KPFM), are widely used for mapping the SP of graphene as well as identification of graphene layers. For example, KPFM has recently been used to distinguish between areas of 1LG, 2LG, few layer graphene (FLG) and the buffer or interfacial layer (0LG) and for validation of optical quality control methods for graphene ${ }^{3,4}$. However, KPFM does not generally provide reliably comparable values for differences in SP between layers with a wide variety of $\Delta V_{C P D}{ }^{1-2 \mathrm{LG}}$ values previously reported for 1-2LG. For example, for epitaxial graphene on SiC, Filleter et al. ${ }^{3}$ reported a $\Delta V_{C P D}{ }^{1-2 \mathrm{LG}}=135 \mathrm{mV}$ in vacuum, whereas Burnett et al. ${ }^{5}$ obtained a $\Delta V_{C P D}{ }^{1-2 \mathrm{LG}}=25 \mathrm{mV}$ in air. On the other hand, in case of exfoliated graphene on $\mathrm{SiO}_{2}$, Yu et al. ${ }^{6}$ reported a $\Delta V_{C P D}{ }^{1-2 \mathrm{LG}}=120 \mathrm{mV}$ after accounting for environmental effects by measuring in ambient atmosphere and dry nitrogen, whereas Ziegler et al. ${ }^{7}$ reported a smaller value of $\Delta V_{C P D}{ }^{1-2 \mathrm{LG}}$ $=68 \mathrm{mV}$ in ambient conditions.

The effects of substrate on the charge transfer to graphene and subsequent change of the SP have been discussed in depth ${ }^{7-10}$. Additionally, change in the charge carrier concentration, whether it is intentional, by electrostatic or photochemical gates ${ }^{11,12}$, or incidental, as by uncontrolled adsorbates, modifies the measured $\Delta V_{C P D}{ }^{1-2 L G}$ values (see ref. 6). For example, specific atmospheric gating can modify the $\Delta V_{C P D}^{1-2 \mathrm{LG}}$ of epitaxial graphene from 0 to $100 \mathrm{mV}$ on changing the environment from vacuum or pure nitrogen to $>1$ ppm NO 2 in nitrogen mixture ${ }^{13}$. Moreover, atmospheric humidity gating has been shown to increase the $\Delta V_{C P D}{ }^{1-2 \mathrm{LG}}$ values ${ }^{14,15}$. While the reported discrepancy in the published values of $\Delta V_{C P D}{ }^{1-2 \mathrm{LG}}$ can be partly attributed to different substrate and environmental gating, here we primarily address the 
measurement methodology and the accuracy of KPFM measurement technique applied to graphene domains.

We analyze the ability to obtain quantified, comparable and accurate results of single-pass frequency-modulated (FM)-KPFM, conventional dual-pass amplitude-modulated (AM)-KPFM and electrostatic force spectroscopy (EFS) by performing measurements on a graphene Hall bar device after SP calibration of the AFM probe against gold electrodes. In contrast to many experimental studies, we aim to investigate graphene devices in standard ambient conditions (rather than in vacuum or specific gas atmosphere), as such conditions are the most representative both for general research and industrial lines. We find that conventional AM-KPFM, being a force sensitive technique, suffers from a spatial averaging effect of the SP due to a significant contribution of the cantilever base and cone to the capacitive coupling, which reduces $\Delta V_{C P D}{ }^{1-2 L G}$ and leads to incorrect values of SP measured on a biased device. In contrast, FM-KPFM is sensitive to the force gradient and measures the SP of the area directly

(a)

AM - KPFM
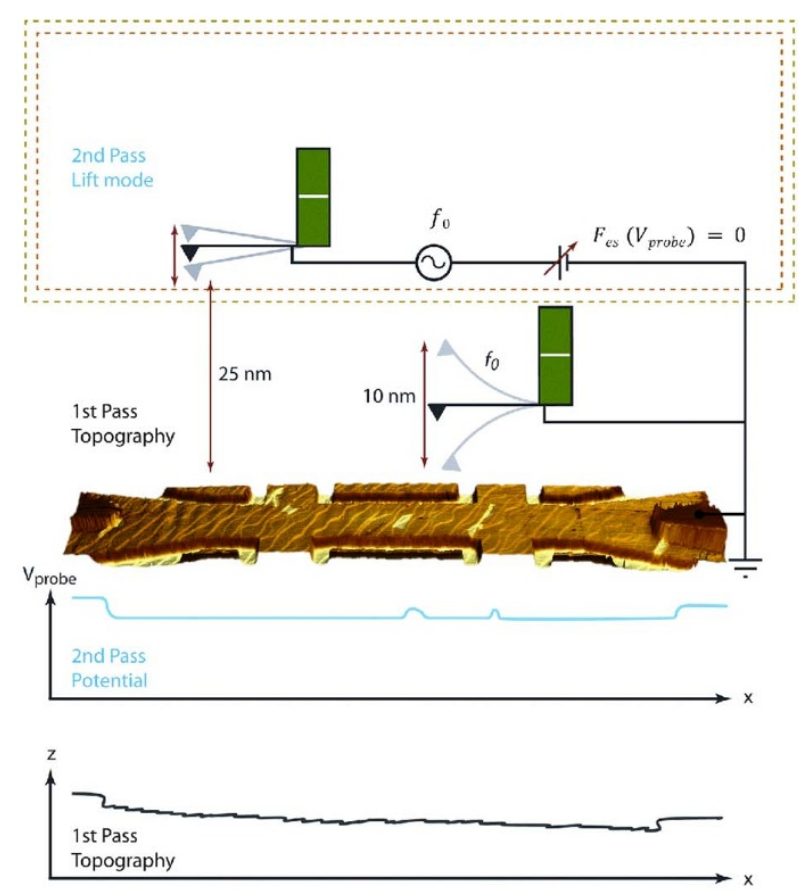

(c)

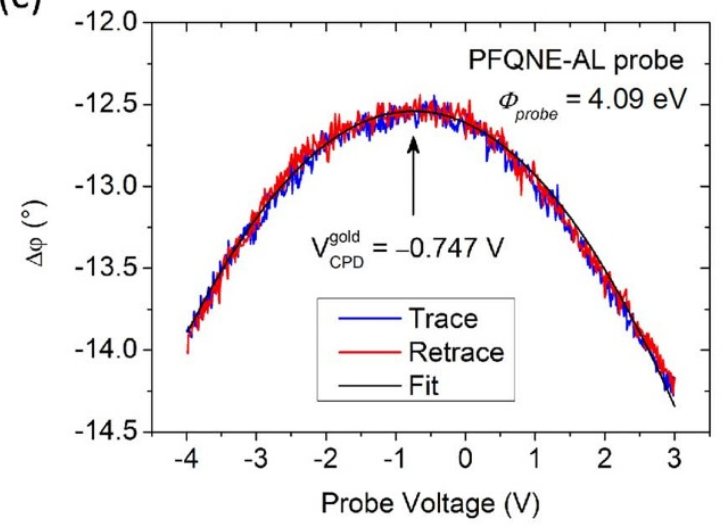

under the probe apex, demonstrating improved spatial resolution and absence of averaging effects. We conclude that FM techniques, such as FM-KPFM and EFS, provide more accurate measurement of the SP than AM-KPFM. Using calibrated FM-KPFM, we perform precise work function measurements of $1 \mathrm{LG}$ and 2LG, being $\Phi_{1 L G}=$ $4.55 \pm 0.02 \mathrm{eV}$ and $\Phi_{2 L G}=4.44 \pm 0.02 \mathrm{eV}$, respectively, for the sample studied here. We also perform contactless measurements of the resistance of the graphene channel and two separate electrodegraphene lead contacts.

We demonstrate that the experimental approach presented here can be successfully used for standardization of measurements of the work function and obtaining reliable quantitative parameters not only in graphene but in many other electronic materials, i.e. semiconductors, photovoltaic, etc. Representing a surface state of a material rather than its bulk property, the work function, in graphene in particular, can be strongly affected by environmental conditions. To assure accuracy of measurements, recalibration

(b)

FM - KPFM
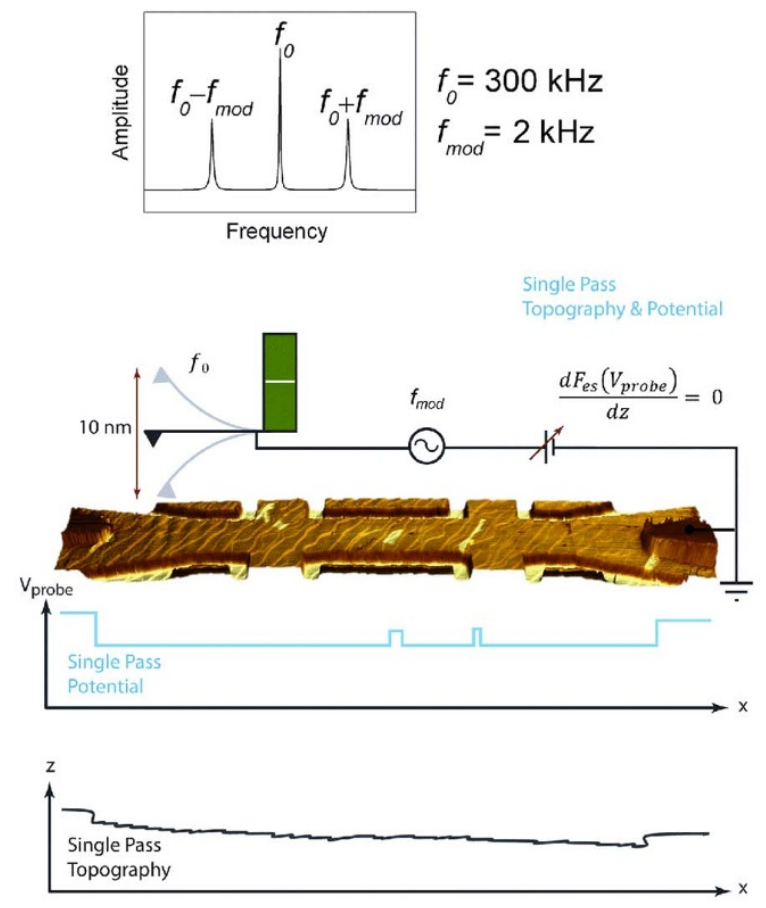

(d)

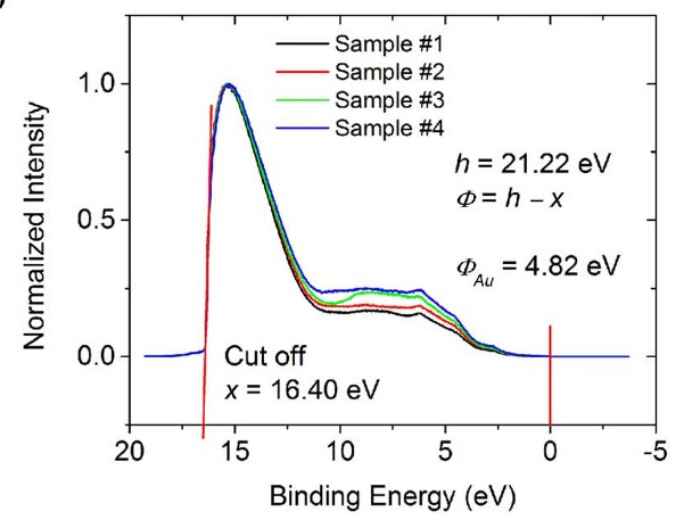

Figure 1 Schematic diagrams of the experimental techniques. (a) AM-KPFM; (b) FM-KPFM; topography of the graphene Hall bar is superimposed with SP maps on a 3D image. Plots show characteristic profiles, i.e. SP on top and topography on bottom along the horizontal line in the center of the image (not shown). (c) Typical parabolic change of the cantilever phase shift measured by EFS during DC voltage sweep at a fixed point on 1LG. (d) UPS data showing the work function of gold, $\Phi_{\mathrm{Au}}=4.82 \mathrm{eV}$, for four different samples. 
should be done upon any significant change of ambient (e.g. humidity).

\section{Results}

Surface potential measurement techniques. Surface potential maps of a sample can be obtained using KPFM, which measures the strength of the electrostatic forces between a conductive probe and the sample ${ }^{5}$. There are different methods of detecting electrostatic forces, namely: AM-KPFM, which responds to the electrostatic force at a set frequency of probe oscillation (Figure 1a); and FM-KPFM, which responds to the electrostatic force, while maintaining constant amplitude of cantilever oscillation (Figure 1b). As we show below, the choice of the measurement technique significantly affects the accuracy of surface potential measurements on micrometer scale graphene. The schematic diagrams of the used techniques are shown in Figure 1; all techniques are discussed in detail in the Method section.

AM-KPFM: experimental results. Figure 2a shows a topography map of the graphene device. SiC step terraces are clearly visible running at a $\sim 60^{\circ}$ angle to the channel. Gold contacts are seen at the left and right hand sides of the image. The image reveals that it is generally rather difficult to determine the graphene layer thickness from the topography maps. The surface potential of the electrically grounded device was mapped using AM-KPFM in ambient environment (Figure 2b). Bright areas of $2 \mathrm{LG}$ are clearly visible on the $1 \mathrm{LG}$ background, whereas darker regions correspond to etched $\mathrm{SiC}$. The value of the $\Delta V_{C P D}{ }^{1-2 \mathrm{LG}}=50 \mathrm{mV}$ is consistently measured over all areas of the sample (Figure 2c), which is comparable to previously published results on similar samples ${ }^{8}$. The SP dip which can be seen to the right of the 2LG step in Figure 2c (as well as in Figure $3 \mathrm{~b}$ and Figure $4 \mathrm{~b}$ ) is attributed to a small patch of resist residue, which is clearly observed in the topography map (circled in red in Figure 2a).

Further to this, we study the surface potential of a biased graphene device. Bias voltages of $V_{c h}=0, \pm 0.5, \pm 1, \pm 1.5$ and $\pm 2 \mathrm{~V}$ were applied to the left gold electrode and SP maps of the device were obtained in AM-KPFM mode. Figure 2d shows the plotted SP values along the marked line (Figure $2 \mathrm{~b}$ ) going through the center of the channel and connecting the gold leads. The raw data is plotted in Figures $2 \mathrm{c}$ and $2 \mathrm{~d}$, i.e. no calibration of the probe's work function has been performed here. As a result, the measured SP values in Figure 2d are not centered at $0 \mathrm{~V}$. A significant discrepancy between applied and measured voltages is observed using AM-KPFM, i.e. the total difference in surface potential values measured on the left gold electrode, when biased with $V_{c h}=+2$ and $-2 \mathrm{~V}$, is only $\sim 2.9 \mathrm{~V}$, i.e. $27.6 \%$ less than the expected $4 \mathrm{~V}$. After taking into account the work function of the probe, the values of $\Delta V_{C P D}$ between the biased gold contacts are still smaller than expected. This discrepancy in applied and measured voltages can be explained by the spatial averaging of AM-KPFM due to the long-range nature of the electrostatic forces acting on the probe and leading to substantial contributions from the probe cone and the base ${ }^{16}$. These parasitic contributions can affect the measured SP, as the area under the cantilever may not be directly over the gold leads, but instead averaging the SP over the graphene device leading to a lower total value. For a given device geometry and using AM-KPFM, it is expected that scanning across the channel might somewhat decrease parasitic capacitive coupling between the cantilever and the gold electrodes ${ }^{16}$. However, bearing in mind that the size of the cantilever $\left(200 \times 30 \mu \mathrm{m}^{2}\right)$ is considerably larger than the device channel $\left(50 \times 5 \mu^{2}\right)$ and intricate device electrode geometry (up to 6 electrodes and bonding pads of a complex shape), some coupling between the cantilever and the gold electrodes and bonding pads is unavoidable in any scanning direction. Moreover,
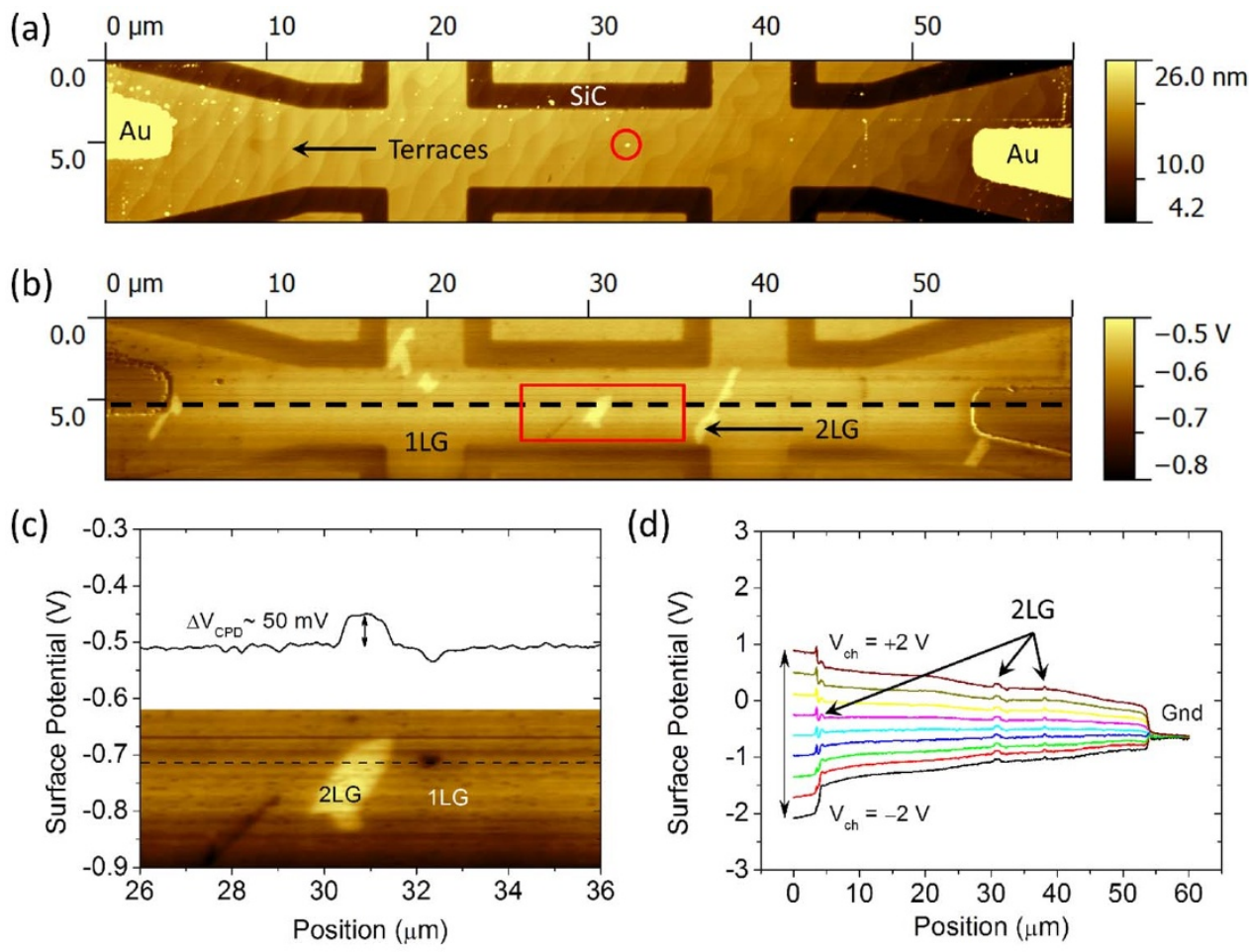

Figure $2 \mid$ Topography and surface potential mapping with AM-KPFM. (a) Topography map of the device showing a double-cross Hall bar, gold electrodes and etched areas (bare SiC) which define the channel. Red circle denotes a small fraction of the resist residue, which is seen as a dip on all SP profiles. (b) AM-KPFM surface potential map of the grounded Hall bar device. (c) Plot of the surface potential between areas of 1LG and 2LG within the channel along the dashed line shown in the inset. Inset shows the magnified area of the AM-KPFM surface potential map framed in (b). (d) Plot of the surface potential for the biased device measured between gold leads through the center of the channel along the line depicted in (b), the left gold lead is biased at $V_{\mathrm{ch}}$ between +2 and $-2 \mathrm{~V}$ and the right gold lead is grounded. 
any inhomogeneity of the device itself (i.e. the presence of graphene domains of different thickness) will contribute to the averaging effect of AM-KPFM technique. It should be noted that the maintaining of absolute uniformity of graphene thickness over the length of $200 \mu \mathrm{m}$ remains challenging.

FM-KPFM: experimental results. Surface potential mapping has been further carried out using FM-KPFM on the same device. Figure $3 \mathrm{a}$ shows the potential map of the grounded device. Areas of $1 \mathrm{LG}$ and $2 \mathrm{LG}$ are sharply outlined and better defined compared to the measurements taken with AM-KPFM. Values of $\Delta V_{C P D}{ }^{1-2 \mathrm{LG}}=$ $150 \mathrm{mV}$ are recorded as shown in Figure $3 \mathrm{~b}$. This value is consistent over the device and significantly larger than $\Delta V_{C P D}{ }^{1-2 L G}$ obtained with AM-KPFM. The larger $\Delta V_{C P D}^{1-2 L G}$ values can be accounted for by considering the measurement technique, which uses the force gradient rather than the force and also leads to improved spatial resolution of FM-KPFM compared to AM-KPFM. Figure 3c shows a line profile of the surface potential measured along the center of the channel with $V_{c h}=0, \pm 0.5, \pm 1, \pm 1.5$ and $\pm 2 \mathrm{~V}$. The change in surface potential values measured on the left gold lead when biased with $V_{c h}=+2$ and $-2 \mathrm{~V}$ is now $\sim 4.18 \mathrm{~V}$, i.e. $4.5 \%$ larger than the expected $4 \mathrm{~V}$, suggesting that this technique provides improved SP measurements even over relatively small structures with a size of several micrometers. This result is in a very good agreement with recent finding ${ }^{17}$, where a negligible averaging effect was demonstrated for graphene samples using FM-KPFM mode.

Electrostatic force microscopy and spectroscopy: experimental results. Figure $4 \mathrm{a}$ shows an EFM phase map of the grounded device. Due to the high spatial resolution, the edges of 2LG domains are sharp and well defined. Figure $4 \mathrm{~b}$ shows the recorded $\mathrm{SP}$ values obtained from EFS measurement points over the area of $1 \mathrm{LG}$ and 2LG. As this is a spectroscopy rather than a mapping technique, values may be slightly affected by the exact position. In this instance, $\Delta V_{C P D}{ }^{1-2 \mathrm{LG}}=110 \mathrm{mV}$ is in a reasonable agreement with the results obtained by FM-KPFM, this being expected as both techniques are sensitive to the force gradient. The discrepancy can be attributed to only a few EFS experimental points obtained on the small isolated 2LG domain at the center of the channel, whereas a significantly larger number of points were measured with FMKPFM. Further improvement of EFS method and better agreement with FM-KPFM can be achieved by decreasing the step size between measurement points. Results of measurements of 200 EFS spectroscopy points taken along the center of the channel between the two gold contacts with the left contact biased at $V_{\mathrm{ch}}=0, \pm 1$ and $\pm 2 \mathrm{~V}$ are shown in Figure 4c.

Even the most accurate FM-KPFM and EFS techniques provide a non-zero reading of the surface potential on the grounded electrode, i.e. $V_{C P D}=-365 \mathrm{mV}$ for FM-KPFM (Figure 3c) and $V_{C P D}=$ $-723 \mathrm{mV}$ for EFS (Figure 4c). This discrepancy is the result of a work function difference between the gold and PFQNE-AL probe. Further to this, we account for the resulting work function difference by subtracting the $V_{c h}$ obtained from the grounded right contact from the experimental value of the $V_{C P D}$, i.e. $\Delta V=V_{C P D}\left(V_{c h}\right)-$ $V_{C P D}(0)$. The procedure was performed using results of all three experimental techniques for the range of applied $V_{c h}$, providing $\Delta V$ for the left gold electrode (Figure 5). The measured potential drop is typically $27.6 \%$ lower than the actual $V_{c h}$ for AM-KPFM, whereas it is $4.4 \%$ and $7.8 \%$ higher for FM-KPFM and EFS, respectively. The lower $\Delta V$ measurements are consistent with spatial averaging, as the relatively large base of the cantilever weakly interacts with the device channel and the right contact ${ }^{16}$, both of which are at a lower $V_{C P D}$ than the left contact, as was discussed above. The higher $\Delta V$ measurement with FM-KPFM could be a result of an overestimation of the SP due to a relatively large excitation voltage of $V_{A C}=8 \mathrm{~V}$, whereas the discrepancy with EFS rises from un-optimized fitting parameters.

Work function calibration. We employed the use of force gradient techniques to provide accurate measurements of work function of $1 \mathrm{LG}$ and 2LG. Initially, work function of the PFQNE-AL probe was calibrated against the work function of the gold leads: $\Phi_{\text {probe }} \approx \Phi_{\mathrm{Au}}$ $+e \Delta V_{\mathrm{CPD}}$, where $V_{\mathrm{CPD}}$ was measured on the grounded gold electrodes. Ultraviolet photoelectron spectroscopy (UPS) measurements were carried out on four separate samples of gold deposited by e-beam evaporation under the same conditions as the deposition of the gold electrodes. The spectra were acquired with voltage of $-19.04 \mathrm{~V}$ applied to the sample. The Fermi edge was centered at $0 \mathrm{eV}$ by measuring the offset from a high resolution Fermi edge spectrum of the silver calibration sample. The offset was used to correct the energy scale for all four Au spectra (Figure 1d). Using the indicated energies obtained from the spectra from each area of
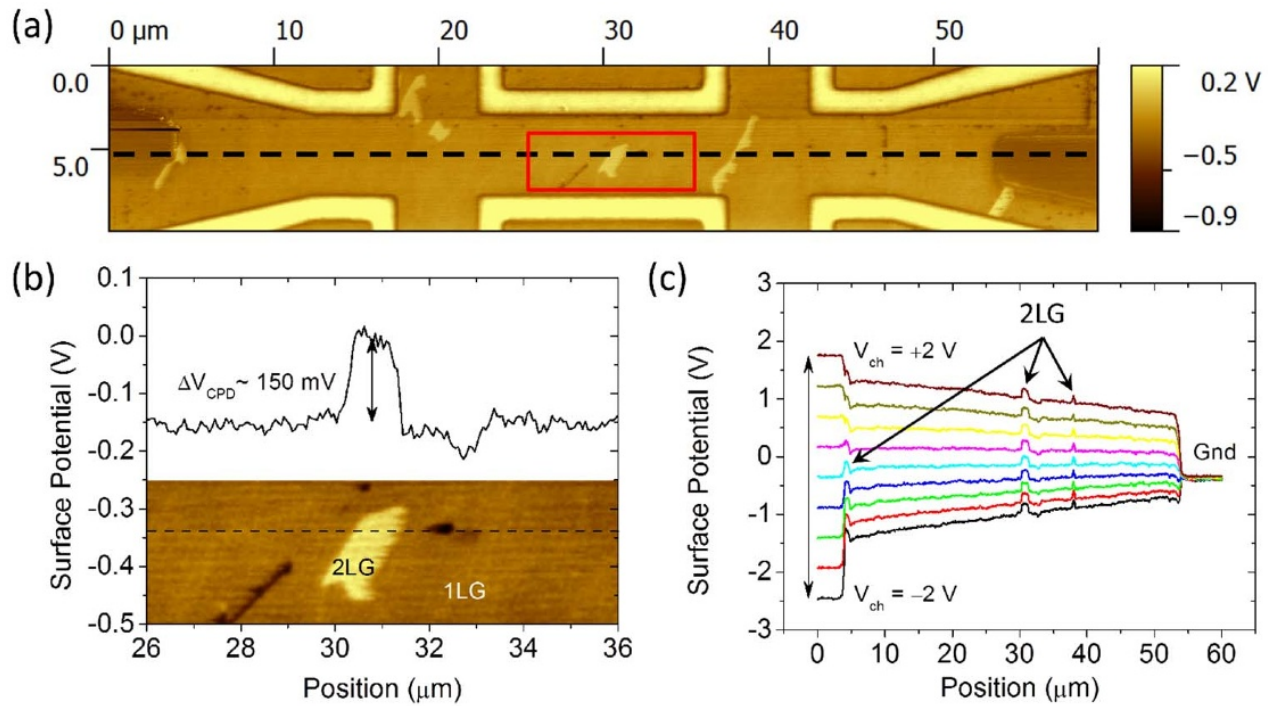

Figure 3 Surface potential mapping with FM-KPFM. (a) FM-KPFM surface potential map of the grounded Hall bar device. (b) Plot of the surface potential between areas of 1LG and 2LG within the channel along the dashed line shown in the inset. Inset shows the magnified area of the FM-KPFM surface potential map framed in (a). (c) Plot of the surface potential measured between gold leads through the center of the channel along the line depicted in (a), the left gold lead is biased at $V_{\mathrm{ch}}$ between +2 and $-2 \mathrm{~V}$ and the right gold lead is grounded. 
(a)

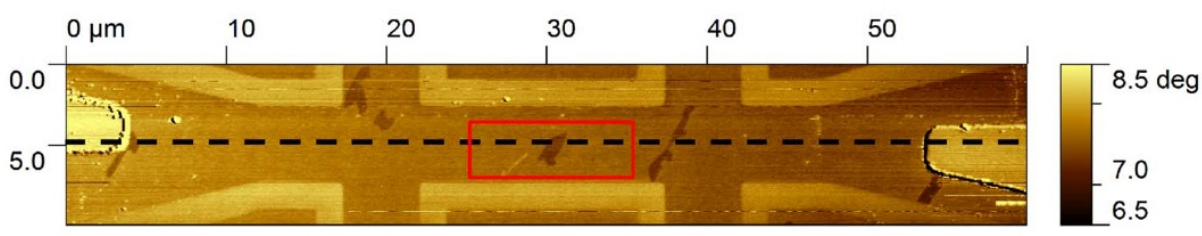

(b)

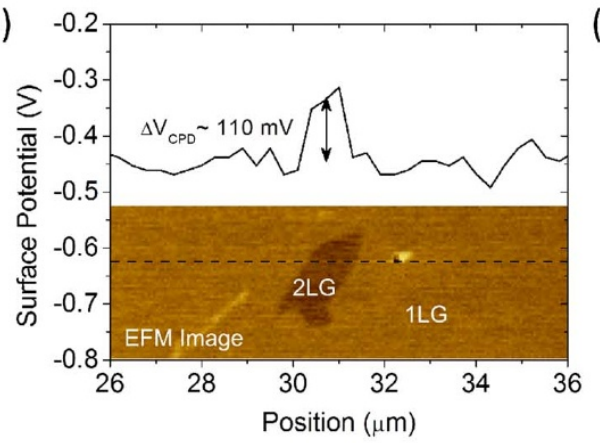

(c)

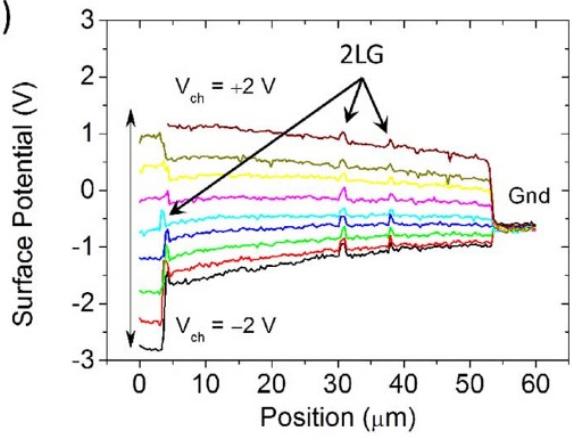

Figure 4 EFM mapping and surface potential line profiles with EFS. (a) EFM phase map of the grounded Hall bar device. (b) EFS plot of the surface potential between areas of 1LG and 2LG within the channel along the dashed line shown in the inset. Inset shows the magnified area of the EFM phase map framed in (a). (c) Plot of the surface potential measured by EFS between gold leads through the center of the channel along the line depicted in (a), the left gold lead is biased at $V_{\mathrm{ch}}$ between +2 and $-2 \mathrm{~V}$ and the right gold lead is grounded.

the samples, the work function was calculated. The difference in energy between the Fermi edge measured on a silver calibration sample and the cut off $(x)$ is given by $x=h-\Phi$, where the energy of the incident photon is $h=21.22 \mathrm{eV}$. The cut off was obtained by fitting a line to the relevant part of each spectrum, determining its gradient and the point at which it crosses the energy-axis. The absolute value of $x=16.40 \mathrm{eV}$ was established, thus measuring the work function of all four gold samples as $\Phi_{\mathrm{Au}}=4.82 \mathrm{eV}$. UPS characterization performed in ultra-high vacuum (UHV) would include all irreversibly bound adsorbates (chemisorbed oxygen and physisorbed hydrocarbon) attached to the surface prior to the measurements. Some uncertainty may arise from the reversible adsorption of species, such as water, which may form surface dipoles leading to a small change of the $\Phi_{\mathrm{Au}}$ on transferring the sample from UHV to ambient environmental conditions. Owing to its relatively high standard electrode potential of $1.52 \mathrm{eV}^{18,19}$, gold is very stable in air, being less prone to both oxidation and formation of a submonolayer of water in ambient conditions (both these factors could potentially affect $\Phi_{\mathrm{Au}}$ ) than most other good conductors. Following Ref. 20, we can estimate that $\Phi_{\mathrm{Au}}$ may decrease by $\sim 3 \%$ (top estimation) as the relative humidity changes from 0 to $40 \%$. Use of a more stable metal would be ideal for tip calibration, however gold remains one of the best electrode materials.

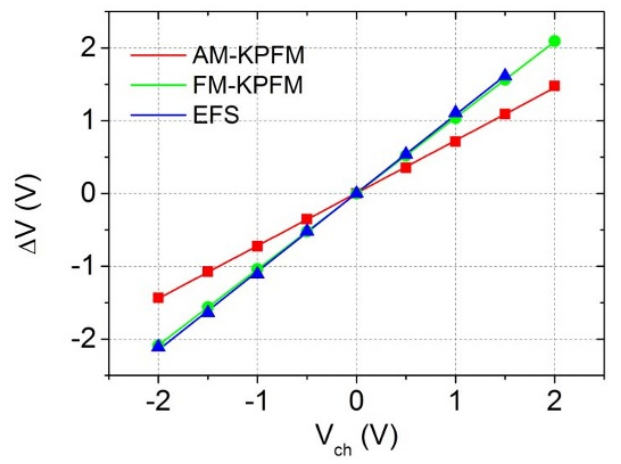

Figure $5 \mid$ Direct comparison of the surface potential measurements. Normalized SP values as measured by AM-KPFM, FM-KPFM and EFS techniques on the left gold electrode in dependence on the voltage applied to the same gold electrode.
Using the measured work function of gold, we calculated the work function of the probe to be $\Phi_{\text {probe }}=4.09 \mathrm{eV}$ using EFS (Figure 1c). Then, the work function of $1 \mathrm{LG}$ and $2 \mathrm{LG}$ were determined: $\Phi_{\text {sample }} \approx$ $\Phi_{\text {probe }}-e \Delta V_{C P D}$ using the measured values of the SP extracted from the line profiles of the potential maps, i.e. $V_{C P D}=-454$ and $-344 \mathrm{mV}$ for $1 \mathrm{LG}$ and $2 \mathrm{LG}$, respectively, see Figure $4 \mathrm{~b}$. This defines work functions of $\Phi_{1 L G} \sim 4.55 \pm 0.02 \mathrm{eV}$ and $\Phi_{2 L G} \sim 4.44 \pm$ $0.02 \mathrm{eV}$. These work function values are within the range of previously reported results of 4.41-4.57 eV for 1LG measured with FM$\mathrm{KPFM}^{6,21}$. It should be noted that the work function of graphene depends on the carrier density and is, therefore exceptionally sensitive to substrate and environmental gating due to its two-dimensional nature. For example, the published values were reported to change with varying lab ambient, i.e. the change of work function and SP due to adsorbates being $<50 \mathrm{meV}^{6}$ and $\sim 130 \mathrm{mV}^{21}$, respectively.

Contactless resistance measurements. High accuracy of FM-KPFM technique provides an excellent contactless method for measuring the electrode-graphene contact resistance with no need for specifically patterned electrodes ${ }^{6}$, which is typically used with the transmission line method. Using experimental results shown in Figure 3c (i.e. line profiles of $V_{C P D}$ at $V_{c h}= \pm 2 \mathrm{~V}$ ), contact and channel resistance can easily be deduced by normalizing these line profiles $\left[V_{C P D}\left(V_{c h}\right)-V_{C P D}(0)\right] / V_{c h}=\Delta V / V_{c h}$ as shown in Figure 6a. This procedure accounts for any intrinsic $V_{C P D}$ changes, i.e. variations in the work function of features, such as $1 \mathrm{LG}, 2 \mathrm{LG}$ and gold. The resulting normalized line profile is solely a consequence of the potential drop at electrode-graphene contacts and along the graphene channel due to changes in the resistance. Dependence of the normalized voltage drop $\Delta V / V_{c h}$ as measured across the left (right) contacts and graphene as well as across the graphene channel (i.e. points 1-2, 3-4 and 2-3, respectively, in Figure 6a) are plotted in Figure $6 \mathrm{~b}$. While the voltage drop within the graphene channel is constant for all applied $V_{c h}$, this value changes linearly on electrode-graphene contacts. Careful inspection of the electrode-graphene potential drop for both contacts reveals a clear $V_{c h}$ dependence. Focusing on the left contact (points $1-2)$, relative change of the voltage on electrode-graphene channel is $\Delta V=0.55$ and $-0.91 \mathrm{~V}$ for $V_{c h}=+2 \mathrm{~V}$ and $-2 \mathrm{~V}$, respectively. However, at the right contact, the $\Delta V=0.83$ and $-0.52 \mathrm{~V}$ for $V_{c h}=$ $+2 \mathrm{~V}$ and $-2 \mathrm{~V}$, respectively. From potential drop and $I-V_{c h}$ 

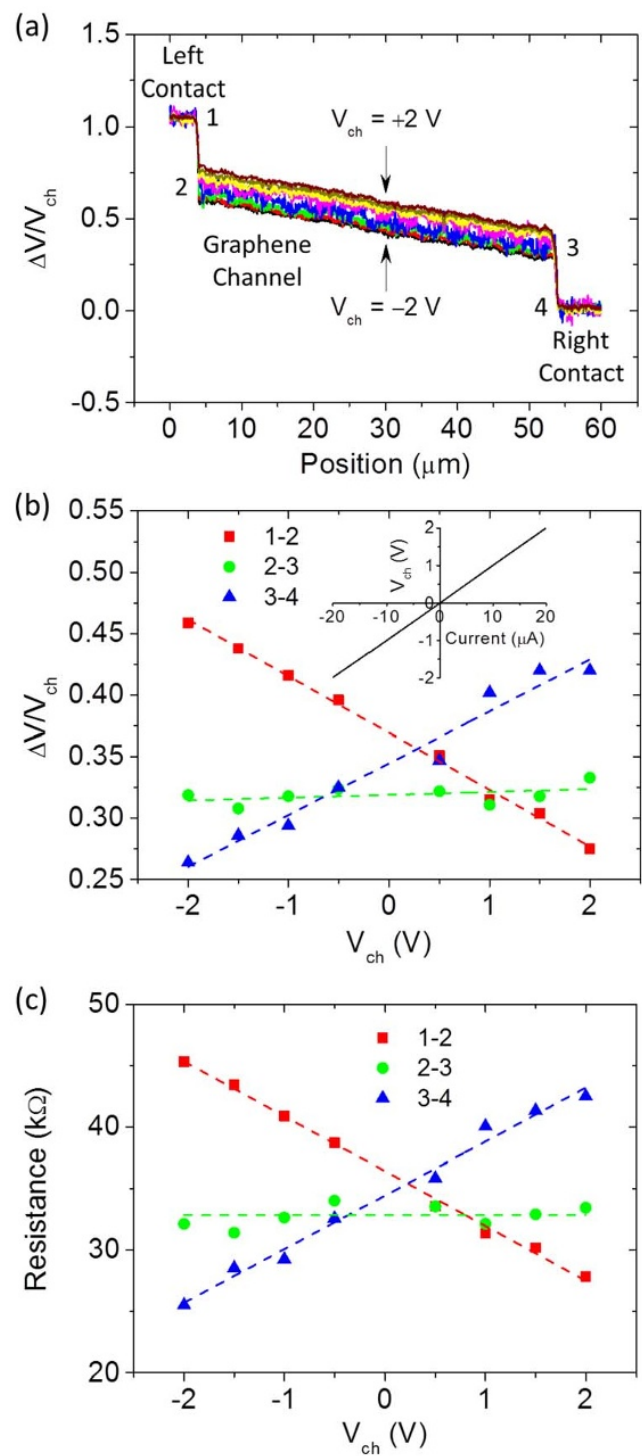

Figure 6 Contactless resistance measurements with FM-KPFM.

(a) Normalized surface potential line profiles. Experimental values are obtained by FM-KPFM along the dashed line in Figure 3a. (b) $\Delta V / V_{c h}$ and (c) resistance measurements of left contact, right contact and across the graphene channel, i.e. points $1-2$, points $3-4$ and points $2-3$, respectively, in (a). Dashed lines are guides for the eye. Inset in (b) shows the dependence of the total current $(I)$ through the circuit on the bias voltage $\left(V_{c h}\right)$.

(transport) measurements (Figure $6 \mathrm{~b}$ main panel and inset, respectively), the contactless resistance can be determined as $\Delta V / I\left(V_{c h}\right)$. Figure $6 c$ shows the contactless resistance measurements of the graphene channel $\left(R_{c h}\right)$ only and electrodegraphene contacts $\left(R_{\text {cont }}\right)$ separately for the left and right contacts. The resistance of the graphene channel $R_{c h} \sim 33 \mathrm{k} \Omega$ is constant over the range of applied voltages, i.e. independent on the $V_{c h}$. The corresponding resistivity value is $\rho_{c h} \sim 2.7 \times 10^{-6} \mathrm{Ohm} \mathrm{cm}$. On the other hand, the contacts exhibit a significant change of $\Delta R_{\text {left }}$ cont $\sim-17.5 \mathrm{k} \Omega$ and $\Delta R_{\text {right cont }} \sim 17.0 \mathrm{k} \Omega$ as $V_{\text {ch }}$ changes from -2 to $+2 \mathrm{~V}$ for left and right contacts, respectively. These measurements show that for this particular device, $R_{\text {cont }}$ is dependent on the $V_{c h}$ revealing a non-Ohmic behavior. It should be noted that the edges of both gold electrodes overlap onto 2LG islands (Figure $3 \mathrm{~b}$ ), which exhibit a lower work function. The lower $\Phi_{2 L G}$ is a result of a high carrier density of the 2LG system, which could affect the flow of charge and, thus the contact resistance. However, it is rather difficult to give a quantitative measure of this effect from our experiments.

\section{Discussion}

Using epitaxial graphene Hall bars with gold electrodes, we have demonstrated significant differences in accuracy and resolution between AM-KPFM and FM-KPFM techniques in determining the difference in surface potential between 1LG and 2LG. Values of $\Delta V_{C P D}{ }^{1-2 \mathrm{LG}}$ measured with FM-KPFM demonstrate a threefold increase as compared to AM-KPFM. While AM-KPFM measures the electrostatic force on the cantilever, FM-KPFM is sensitive to the force gradient. Thus, AM-KPFM gives a weighted average of the signal, including contributions from the surface under the probe cone and cantilever. Sensitivity to the shorter range force gradient characteristic for FM-KPFM leads to spatially-confined contributions, which arise only from the probe apex, thereby reducing the spatial averaging of measured work functions observed in AMKPFM. We experimentally demonstrate that FM-KPFM and consequently EFS have a greater degree of spatial resolution $(<20 \mathrm{~nm})$ than AM-KPFM. Improvement in spatial resolution is clear on comparing the sharpness of the potential maps obtained. Moreover, we show that use of FM-KPFM and a calibrated probe provide a simple and straightforward method of obtaining an accurate measure of the work function of 1LG and 2LG. Accuracy of measurements is provided by initial calibration of the KPFM signal against the gold electrodes, whose work function was measured independently by UPS, however, keeping in mind that the work function of gold may somewhat change on transferring the sample to ambient due to adsorption of reversible species as discussed above. This improvement in measurement technique enables greater accuracy in determination of the work function of $1 \mathrm{LG}$ and $2 \mathrm{LG}$, with values of $\Phi_{1 L G} \sim 4.55 \pm$ $0.02 \mathrm{eV}$ and $\Phi_{2 L G} \sim 4.44 \pm 0.02 \mathrm{eV}$, respectively, as valid for the particular sample studied here and in specified ambient conditions. Thus, the experimental procedure outlined in this paper can be implemented as a generic route for standardization of work function measurements applicable not only to graphene but to a wide class of nanoscale materials and devices, for example in light emission and solar devices, displays, etc.

FM-KPFM was also used to investigate: i) the contact resistance between the gold electrode and graphene, revealing a non-Ohmic behavior, and ii) the resistance of the graphene channel showing Ohmic behavior with $R_{c h} \sim 33 \mathrm{k} \Omega$ and $\rho_{c h} \sim 2.7 \times 10^{-6} \mathrm{Ohm}$ $\mathrm{cm}$. This simple contactless method can be used to investigate the specific components of the total resistance, without fabricating devices for the transmission line method.

Our results unambiguously demonstrate that the measured values of the SP of single- and bi-layer graphene largely depend on the accuracy of the technique. However, we would like to stress that the obtained absolute values are not by any means the fundamental parameters for graphene and are strongly dependent on the state of the surface. The carrier concentration and correspondently the work function of graphene are exceptionally sensitive to substrate (intrinsic) and environmental (extrinsic) gating due to its twodimensional nature. Being representative of the surface state, the work function can be strongly affected by such factors as traces of gas contamination, temperature and, in particular, humidity. For example, by conducting experiments in the controllable humidity (outside of the scope of the present paper) we found that standard $(\sim 10-15 \%)$ variations corresponding to typical day-to-day change of the lab humidity, led to a corresponding change of $\Delta V_{C P D}{ }^{1-2 \mathrm{LG}} \sim$ 25-30 mV. Moreover, it was reported that $1 \mathrm{LG}$ and 2LG have different adsorption energies for gases, such as $\mathrm{NO}_{2}{ }^{22}$ and water vapour ${ }^{15}$, which leads to the differences in the doping levels for these two domains. Thus, without reproducing substrate and environmental conditions, the SP and work functions of graphene 
obtained in different experiments cannot be adequately compared. This places an even stronger emphasis on the necessity of standardization in the measurements of the SP and work function for each particular sample of interest and for given environmental conditions.

\section{Methods}

Sample preparation. Nominally monolayer epitaxial graphene was prepared by sublimation of $\mathrm{Si}$ and the subsequent graphene formation on the Si-terminated face of an on-axis $4 \mathrm{H}-\mathrm{SiC}(0001)$ substrate at $2000^{\circ} \mathrm{C}$ and 1 bar argon gas pressure. Details of the growth and structural characterization are reported elsewhere ${ }^{2}$. The specific synthesis route has been developed to provide large areas of homogeneous singlelayer graphene. The resulting material is $\mathrm{n}$-doped, owing to charge transfer from the interfacial layer ${ }^{23,24}$, with the measured electron concentration in the range $n=6-20$ $\times 10^{11} \mathrm{~cm}^{-2}$ and carrier mobility of $\mu \sim 3000 \mathrm{~cm}^{2} \mathrm{~V}^{-1} \mathrm{~s}^{-1}$ at room temperature ${ }^{25,26}$. The epitaxial graphene device was fabricated by electron beam lithography (PMMA/MMA and ZEP520 resists), oxygen plasma etching and evaporation of Ti/ $\mathrm{Au}(5 / 100 \mathrm{~nm})$ electrodes. Details of the sample fabrication are reported elsewhere ${ }^{26}$. The device comprises two crosses with a channel width of $4.8 \mu \mathrm{m}$, surrounded by $1.6 \mu \mathrm{m}$-wide trench etched down into the $\mathrm{SiC}$ substrate. The transport measurements were performed in air, at room temperature, in a dark environment. Details of the measurements are reported in Ref. 26.

Standard lithography fabrication methods lead to a thin (1-2 $\mathrm{nm}$ ) layer of a resist residue on top of the graphene. The residues can significantly affect the carrier density and even type, as shown in our previously published work in this area ${ }^{27}$. For instance, we show exposure of the resist residue to 250 -nm wavelength UV light for 20 minutes leads p-doping of the graphene, where $n_{h}=2.5 \times 10^{12} \mathrm{~cm}^{-2}$ with $\Phi_{1 L G}=4.68 \mathrm{eV}$. Subsequently, cleaning the residues using contact-mode AFM restored the n-type conduction of the graphene, where $n_{e}=1.17 \times 10^{12} \mathrm{~cm}^{-2}$ with $\Phi_{1 L G}=4.35 \mathrm{eV}$. To avoid resist related doping, the device was cleaned by sweeping away the residual resist and partly atmospheric adsorbates from the surface using contact-mode AFM prior to imaging. In order to avoid permanent damage to the device, soft contactmode cantilevers (Bruker) with a set point of $\sim 40 \mathrm{nN}$ was used.

SPM measurements. SP measurements were carried out in ambient environment at a controlled temperature of $18^{\circ} \mathrm{C}$ and humidity of $\sim 35 \%$. The measurements were conducted on a Bruker Dimension Icon SPM. Doped silicon PFQNE-AL probes (Bruker) with a probe radius of $\sim 5 \mathrm{~nm}$ and a spring constant of $\sim 0.8 \mathrm{~N} / \mathrm{m}$ were used for electrical measurements. Topography height images of the graphene device were recorded simultaneously with tapping phase and SP maps were compiled from either AM-KPFM, FM-KPFM or EFM phase shift.

Electrostatic force microscopy. Electrostatic force microscopy (EFM) is performed as a dual-pass technique: first, the topography line profile is recorded in tapping mode, and then the line profile is traced at a set lift height above the surface. During the second lifted pass, the cantilever is mechanically oscillated at $f_{0}$, while a constant DC bias $\left(V_{D C}\right)$ is applied, probing the probe-sample electrostatic forces, which depend on the probe-sample capacitance $C$ and height $z^{28}$ :

$$
F_{D C}=\frac{1}{2} \frac{d C}{d z} V^{2}, \text { where } V=V_{C P D}+V_{D C}+V_{\text {induced }} .
$$

EFM is a purely DC technique. The electrostatic forces affect the amplitude, resonant frequency and phase of the cantilever oscillation. The EFM image is generated by recording the cantilever phase changes with a lock-in amplifier

$$
\Delta \varphi=\frac{Q}{k} \frac{d F_{D C}}{d z}=\frac{Q}{2 k}\left(\frac{d^{2} C}{d z^{2}}\right) V^{2},
$$

where $k$ is the spring constant and $Q$ is quality factor of the cantilever. The EFM technique operates on the force gradient $\left(d F_{D C} / d z\right)^{29}$, giving sharper contrast between areas of different electrical properties. Being confined to the probe apex, the force gradient decays much faster with distance than the force itself and, therefore it is less affected by the parasitic capacitance of the cantilever base. However, EFM provides only qualitative information on the electronic properties of sample surface, as the individual voltage components are not separated ${ }^{29}$.

Electrostatic force spectroscopy. Electrostatic force spectroscopy (EFS) is performed at points of interest defined by EFM or other mapping techniques. Each measurement consists of oscillating the probe at $f_{0}$, while sweeping $V_{\text {probe }}$ and simultaneously recording $\Delta \varphi$. The plots of $\Delta \varphi$ as a function of $V_{\text {probe }}$ are parabolic, where the inflection point of the parabola is the point at which $d F_{D} / d z$ is nullified, i.e. the force on the probe is zero (Figure 1c). The inflection point is extracted post measurement and the resulting $V_{\text {probe }}$ at which $d F_{D C} / d z=0$ defines the surface potential. EFS spectroscopy was conducted along the center of the device channel, i.e. 200 spectroscopy points were taken on the graphene channel along the marked line connecting the gold leads with the step of $\sim 300 \mathrm{~nm}$ between individual points. The step between individual points can be significantly decreased with the restricting factor being the lateral step resolution of the SPM system, which is generally limited by the diameter of the probe apex. Thus, the step wise change $<20 \mathrm{~nm}$ in the surface potential at $1-2 \mathrm{LG}$ interface can be readily observed ${ }^{30}$.
EFS can be used as a quantitative and accurate measure of the SP and $\Phi_{\text {sample }}$ of a sample, if the probe is first calibrated against a sample of known $\Phi$. As EFS is not a scanning technique, probe degradation and the relevant work function change are negligible. EFS could be performed at every point of a two dimensional raster if time is not a constraint.

Calibrated work function measurements of graphene were obtained with EFS by calibrating the work function of the probe against the known work function of gold electrodes, which was measured by ultraviolet photoemission spectroscopy (UPS), see Figure 1d.

Amplitude-modulated KPFM. The AM-KPFM, discussed here, is performed as a dual-pass technique; topography line profile is mapped with tapping mode AFM during the first pass, which is then traced at a set lift height above the surface performing the surface potential measurement (Figure 1a). During the second pass of AM-KPFM, the mechanical drive to the cantilever is disabled and an AC bias voltage $\left(V_{A C}=2 \mathrm{~V}\right)$ is applied to the probe at the mechanical resonance $f_{0}$ of the cantilever. The $V_{A C}$ causes the cantilever to oscillate due to the attractive and repulsive electrostatic interaction $\left(F_{e s}\right)$ between the probe and the sample ${ }^{31}$

$$
F_{e s}=-\frac{1}{2} \frac{d C}{d z}\left[\left(V_{D C}-V_{C P D}\right)+V_{A C} \sin (\omega t)\right]^{2}
$$

where $V_{D C}$ is a DC bias voltage and $V_{C P D}$ is a contact potential difference between the probe and sample. A proportional-integral-derivative (PID) feedback loop monitors and minimizes the amplitude of the cantilever oscillations by applying a

compensating $V_{D C}$ to the probe to cancel the probe-sample electrostatic forces, i.e. $V_{D C}=V_{C P D}$ is maintained at each pixel. The applied $V_{D C}$ is recorded at each point, providing a map of the SP. This conventional dual-pass KPFM is a well-established technique, widely used for quantitative probing of the surface potential of graphene $e^{1,3,6,32,33}$. Generally, the technique suffers from a poor lateral resolution, $\sim 50-70 \mathrm{~nm}$, see e.g. Ref. 31 .

Frequency-modulated KPFM. FM-KPFM, discussed here, is a single-pass technique, which gives a greater degree of spatial resolution than AM-KPFM as it measures the force gradient $\left(d F_{e s} / d z\right)^{29}$ rather than the force acting on the entire cantilever. The probe-sample electrostatic forces affect the resonance frequency of the cantilever, where the amplitude $(A)$ of the cantilever excitation at $f_{0} \pm f_{\text {mod }}$ depends on the electrostatic force in the system

$$
A\left(f_{0} \pm f_{\mathrm{mod}}\right) \approx f_{0}\left(1-\frac{1}{2 k} \frac{d F_{e s}}{d z}\right),
$$

where $k$ is the spring constant of the probe. The topography is determined with the tapping mode at the cantilever resonance, $f_{0} \approx 300 \mathrm{kHz}$. Simultaneously, a lower frequency $\left(f_{\text {mod }} \approx 2 \mathrm{kHz}\right)$ AC voltage $\left(V_{\mathrm{AC}}=8 \mathrm{~V}\right)$ is applied to the cantilever. This modulation results in the appearance of side lobes in the cantilever oscillation spectrum at frequencies $f_{0} \pm f_{\text {mod }}$ (Figure 1b). The FM-KPFM PID feedback loop minimizes the side lobes by applying a compensating $V_{D C}$ at each pixel. In a similar fashion to AM-KPFM, the probe-sample electrostatic forces are nullified when $V_{D C}=$ $V_{C P D}$, therefore recording $V_{D C}$ and generating the SP map. However, in contrast to AM-KPFM, FM-KPFM typically requires stiffer, higher frequency cantilevers. FMKPFM offers a higher spatial resolution of $<20 \mathrm{~nm}$ as a result of force gradient localized to the probe apex and higher sensitivity to frequency shifts ${ }^{29}$.

While AM-KPFM is usually performed as a dual-pass technique where first topography and then SP are measured along the same line in an alternating fashion, FM-KPFM is most often performed as a single-pass technique, where topography and potential are recorded simultaneously, thus improving the speed of image capture. However, it should be noted that being either single- or dual-pass is not a definition of the techniques, as other examples have been demonstrated previously ${ }^{34,35}$.

1. Emtsev, K. V. et al. Towards wafer-size graphene layers by atmospheric pressure graphitization of silicon carbide. Nature Materials 8, 203-7 (2009).

2. Yakimova, R. et al. Analysis of the Formation Conditions for Large Area Epitaxial Graphene on SiC Substrates. Materials Science Forum 645-648, 565-568 (2010).

3. Filleter, T., Emtsev, K. V., Seyller, T. \& Bennewitz, R. Local work function measurements of epitaxial graphene. Applied Physics Letters 93, 133117 (2008).

4. Yager, T. et al. Nano Lett. Nano Lett. Article ASAP; DOI: 10.1021/nl402347g (2013).

5. Burnett, T., Yakimova, R. \& Kazakova, O. Mapping of local electrical properties in epitaxial graphene using electrostatic force microscopy. Nano Letters 11, 2324-8 (2011).

6. Yu, Y.-J. et al. Tuning the graphene work function by electric field effect. Nano Letters 9, 3430-4 (2009).

7. Ziegler, D. et al. Variations in the work function of doped single- and few-layer graphene assessed by Kelvin probe force microscopy and density functional theory. Physical Review B 83, 235434 (2011).

8. Eriksson, J. et al. The influence of substrate morphology on thickness uniformity and unintentional doping of epitaxial graphene on SiC. Applied Physics Letters 100, 241607 (2012)

9. Eriksson, J., Puglisi, D., Vasiliauskas, R., Lloyd Spetz, A. \& Yakimova, R. Thickness Uniformity and Electron Doping in Epitaxial Graphene on SiC. Materials Science Forum 740-742, 153-156 (2013). 
10. Riedl, C., Coletti, C. \& Starke, U. Structural and electronic properties of epitaxial

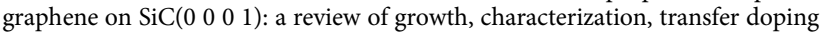
and hydrogen intercalation. Journal of Physics D: Applied Physics 43, 374009 (2010).

11. Lara-Avila, S. et al. Non-volatile photochemical gating of an epitaxial graphene/ polymer heterostructure. Advanced Materials 23, 878-82 (2011).

12. Tzalenchuk, A. et al. Engineering and metrology of epitaxial graphene. Solid State Communications 151, 1094-1099 (2011).

13. Pearce, R. et al. On the Differing Sensitivity to Chemical Gating of Single and Double Layer Epitaxial Graphene Explored Using Scanning Kelvin Probe Microscopy. ACS Nano 7, 4647-4656 (2013).

14. Kazakova, O., Burnett, T. L., Patten, J., Yang, L. \& Yakimova, R. Epitaxial graphene on $\mathrm{SiC}(0001)$ : functional electrical microscopy studies and effect of atmosphere. Nanotechnology 24, 215702 (2013).

15. Burnett, T. L., Patten, J. \& Kazakova, O. Water desorption and re-adsorption on epitaxial graphene studied by SPM. arXiv 20 (2012). at http://arxiv.org/abs/ 1204.3323.

16. Charrier, D. S. H., Kemerink, M., Smalbrugge, B. E., de Vries, T. \& Janssen, R. A. J. Real versus measured surface potentials in scanning Kelvin probe microscopy. ACS Nano 2, 622-6 (2008).

17. Cohen, G. et al. Reconstruction of surface potential from Kelvin probe force microscopy images. Nanotechnology 24, 295702 (2013).

18. Lu, X., Minari, T., Kumatani, A., Liu, C. \& Tsukagoshi, K. Effect of air exposure on metal/organic interface in organic field-effect transistors. Applied Physics Letters 98, 243301 (2011).

19. Bard, A. J., Parsons, R. \& Jordan, J. Standard Potentials in Aqueous Solutions. (Marcel Dekker, New York, 1985).

20. Guo, L. Q., Zhao, X. M., Bai, Y. \& Qiao, L. J. Water adsorption behavior on metal surfaces and its influence on surface potential studied by in situ SPM. Applied Surface Science 258, 9087-9091 (2012).

21. Bussmann, B. K., Ochedowski, O. \& Schleberger, M. Doping of graphene exfoliated on SrTiO3. Nanotechnology 22, 265703 (2011).

22. Pearce, R. et al. Towards optimisation of epitaxially grown graphene based sensors for highly sensitive gas detection. in IEEE Sensors 898-902 (IEEE, 2010). doi:10.1109/ICSENS.2010.5690879.

23. Riedl, C., Coletti, C., Iwasaki, T., Zakharov, A. A. \& Starke, U. Quasi-FreeStanding Epitaxial Graphene on $\mathrm{SiC}$ Obtained by Hydrogen Intercalation. Physical Review Letters 103, 246804 (2009).

24. Janssen, T. J. B. M. et al. Anomalously strong pinning of the filling factor $v=2$ in epitaxial graphene. Physical Review B 83, 233402 (2011).

25. Tzalenchuk, A. et al. Towards a quantum resistance standard based on epitaxial graphene. Nature Nanotechnology 5, 186-9 (2010).

26. Panchal, V. et al. Small epitaxial graphene devices for magnetosensing applications. Journal of Applied Physics 111, 07E509 (2012)

27. Kazakova, O., Panchal, V. \& Burnett, T. Epitaxial Graphene and Graphene-Based Devices Studied by Electrical Scanning Probe Microscopy. Crystals 3, 191-233 (2013)
28. Girard, P. Electrostatic force microscopy: principles and some applications to semiconductors. Nanotechnology 12, 485 (2001)

29. Zerweck, U., Loppacher, C., Otto, T., Grafström, S. \& Eng, L. Accuracy and resolution limits of Kelvin probe force microscopy. Physical Review B 71, 12542 (2005).

30. Panchal, V. et al. Surface potential variations in epitaxial graphene devices investigated by Electrostatic Force Spectroscopy. 2012 12th IEEE Conference on Nanotechnology (IEEE-NANO) 1-5 (2012). doi:10.1109/NANO.2012.6322049.

31. Melitz, W., Shen, J., Kummel, A. C. \& Lee, S. Kelvin probe force microscopy and its application. Surface Science Reports 66, 1-27 (2011).

32. Burnett, T. L., Yakimova, R. \& Kazakova, O. Identification of epitaxial graphene domains and adsorbed species in ambient conditions using quantified topography measurements. Journal of Applied Physics 112, 054308 (2012).

33. Connolly, M. R. \& Smith, C. G. Nanoanalysis of graphene layers using scanning probe techniques. Philosophical Transactions. Series A, Mathematical, physical, and engineering sciences 368, 5379-89 (2010).

34. Ziegler, D. \& Stemmer, A. Force gradient sensitive detection in lift-mode Kelvin probe force microscopy. Nanotechnology 22, 075501 (2011).

35. Glatzel, T., Sadewasser, S. \& Lux-Steiner, M. C. Amplitude or frequency modulation-detection in Kelvin probe force microscopy. Applied Surface Science 210, 84-89 (2003).

\section{Acknowledgments}

This work has been funded by NMS under the IRD Graphene Project (NPL) and EU FP7 Project 'ConceptGraphene'. We are very grateful to Karin Cedergren for help with the nanofabrication of graphene devices and Steve Spencer for UPS measurements. We are grateful to Bruker Nano UK team for constant support of our SPM measurements.

\section{Author contributions}

O.K. designed the research, R.Y. grew the samples, V.P. fabricated nanodevices, V.P. and R.P. performed the measurements, V.P., A.T. and O.K. analyzed the data. All authors discussed the results, participated in writing and commented on the manuscript. All authors reviewed the manuscript

\section{Additional information}

Competing financial interests: The authors declare no competing financial interests.

How to cite this article: Panchal, V., Pearce, R., Yakimova, R., Tzalenchuk, A. \& Kazakova, O. Standardization of surface potential measurements of graphene domains. Sci. Rep. 3 , 2597; DOI:10.1038/srep02597 (2013).

(c) (i) (2) This work is licensed under a Creative Commons Attributioncc. visit http://creativecommons.org/licenses/by-nc-sa/3.0 